\title{
Association between kidney function, nutritional status and anthropometric measures in older people
}

\section{The Screening for CKD among Older People across Europe (SCOPE) study}

Agnieszka Guligowska', Andrea Corsonello ${ }^{2,3}$, Małgorzata Pigłowska', Regina Roller-Wirnsberger ${ }^{4}$, Gerhard Wirnsberger ${ }^{4}$, Johan Ärnlöv ${ }^{5,6,7}$, Axel C. Carlsson ${ }^{5,7}$, Lisanne Tap ${ }^{8}$, Francesco Mattace-Raso ${ }^{8}$, Francesc Formiga ${ }^{9}$, Rafael Moreno-Gonzalez ${ }^{9}$, Ellen Freiberger ${ }^{10}$, Cornel Sieber ${ }^{10}$, Pedro Gil Gregorio ${ }^{11}$, Sara Laínez Martínez ${ }^{11}$, Rada Artzi-Medvedik ${ }^{12}$, llan Yehoshua ${ }^{13}$, Paolo Fabbietti ${ }^{3 *}$ (D) Fabrizia Lattanzio ${ }^{2}$, Tomasz Kostka ${ }^{1}$ and on behalf of SCOPE investigators

\begin{abstract}
Background: Different mechanisms connect the nutritional status with the occurrence and the course of chronic kidney disease (CKD). The end-stage renal disease is complicated by catabolic inflammatory reactions and cachexia which leads to malnutrition (undernutrition). On the other hand, obesity is an important risk factor for the development and acceleration of CKD.

Methods: In the SCOPE study, community-dwelling persons aged 75 years and over, from 6 European countries and Israel were examined at the baseline phase. We assessed the relationship between anthropometric measures (Body Mass Index (BMI), circumferences of arm (AC), waist (WC), hip (HC), and calf (CC), waist-to-hip ratio - WHR, waist-to-height ratio - WHtR, risk of malnutrition (Mini Nutritional Assessment - MNA), serum albumin) and estimated glomerular filtration rate (eGFR) calculated by Berlin Initiative Study (BIS) equation.

* Correspondence: p.fabbietti@inrca.it

${ }^{3}$ Laboratory of Geriatric Pharmacoepidemiology and Biostatistics, IRCCS

INRCA, Via S. Margherita 5, 60124 Ancona, Italy

Full list of author information is available at the end of the article

\section{$\mathrm{BMC}$}

C The Author(s). 2020 Open Access This article is licensed under a Creative Commons Attribution 4.0 International License, which permits use, sharing, adaptation, distribution and reproduction in any medium or format, as long as you give appropriate credit to the original author(s) and the source, provide a link to the Creative Commons licence, and indicate if changes were made. The images or other third party material in this article are included in the article's Creative Commons licence, unless indicated otherwise in a credit line to the material. If material is not included in the article's Creative Commons licence and your intended use is not permitted by statutory regulation or exceeds the permitted use, you will need to obtain permission directly from the copyright holder. To view a copy of this licence, visit http://creativecommons.org/licenses/by/4.0/ The Creative Commons Public Domain Dedication waiver (http://creativecommons.org/publicdomain/zero/1.0/) applies to the data made available in this article, unless otherwise stated in a credit line to the data. 
(Continued from previous page)

Results: We studied 2151 subjects (932 men and 1219 women) with a mean age of $79.5 \pm 5.9$ years. A total of 1333 (62\%) participants had CKD (GRF $<60 \mathrm{ml} / \mathrm{min} / 1.73 \mathrm{~m}^{2}$ ). Negative correlations between eGFR and weight, AC, WC, HC, CC, BMI, WHtR were observed. Positive correlation occurred between eGFR and MNA score (Spearman's rho = 0.11) and albumin concentration (rho =0.09). Higher weight, AC, WC, HC, CC, BMI and WHtR increased the odds ratio of $C K D$; higher $M N A(O R=0.98,95 \% \mathrm{Cl} 0.94-1.0)$ and higher serum albumin $(\mathrm{OR}=0.73,95 \% \mathrm{Cl} 0.53-1.0)$ were weakly associated with reduced odds. The risk of malnutrition was the highest with eGFR $<30$ as compared to $\mathrm{eGFR}>60(\mathrm{OR}=2.95,95 \% \mathrm{Cl}=1.77-4.94$ for $\mathrm{MNA}<24 ; \mathrm{OR}=5.54,95 \% \mathrm{Cl}=1.66-18.5$ for hypoalbuminemia $<3.5 \mathrm{~g} /$ $\mathrm{dL})$.

Conclusion: The population of community dwelling people aged 75+ with CKD shows general features of overweight and obesity with a small prevalence of malnutrition. For anthropometric measures, the strongest association with eGFR and the highest odds of CKD were identified using WC, HC, CC and WHtR. Albumin level and MNA, but not MNA Short Form, indicated an increased odds of malnutrition with a decrease in eGFR.

Keywords: Aging, Chronic kidney disease, MNA, Malnutrition, Undernutrition, Overweight, Obesity

\section{Background}

In recent years, an increase in the prevalence of chronic kidney disease (CKD) has been observed [1]. Currently, in the European population, about $18 \%$ of citizens suffer from CKD [2]. Similarly, the prevalence of obesity in the worldwide population aged 65 years and older is between 18 and $30 \%[3,4]$. Not only obesity affects the elderly, but more and more attention is also paid to the occurrence of malnutrition or undernutrition [5]. Depending on the criterion used and setting, it occurs in about $10 \%$ among independent people up to more than $60 \%$ in rehabilitation hospitals and intensive care units [3].

Nutritional status defined as "the condition of the body in those respects influenced by the diet; the levels of nutrients in the body and the ability of those levels to maintain normal metabolic integrity" [6] contributes to the older people's health status and risk of many diseases. In older age this status is influenced by the cumulative effect of comorbidities, functional status, medical history and economic status. Hormonal, metabolic and other changes may affect the nutritional status of the elderly, predisposing to deviations from normality.

While the association between CKD and nutritional disorders in the general population is well known [7], there is a substantial lack of evidence with regard to older people (i.e. the population more exposed to the burden of both CKD and malnutrition) [8-10]. CKD may be associated with relevant changes in appetite and taste perception, as well as impaired thirst mechanism and increased risk of dehydration [11]. All these changes may increase the risk of malnutrition [12], which in turn may lead to functional impairment [13, 14], sarcopenia and frailty [15], increased risk of falls [16], hospitalization and mortality [17, 18]. On the other hand, age-related hormonal changes together with lower physical activity may promote obesity in the older adults [19, 20]. Excessive body fat, especially visceral, is associated with metabolic disorders and increased inflammation [21], CKD, hypertension and diabetes [22, 23].

In the Screening for Chronic Kidney Disease Among Older People Across Europe (SCOPE) project a lot of attention has been paid to the assessment of participants' nutritional status [24]. The collected data allows reliable and detailed analysis of the relationship between kidney function and the nutritional status. The aim of our study was to assess the relationship between anthropometric measures, malnutrition and kidney function among older adults aged 75 or more. Given that appropriate management of older patients with CKD may contribute to slower loss of kidney function (potentially preventing kidney failure) and enable to control better over its consequences $[25,26]$, understanding the cross-sectional interactions between anthropometric measures, nutritional status and CKD represents a relevant step in prevention strategies.

\section{Methods \\ Design of the study and study population}

The SCOPE study (European Grant Agreement no. 436849), is a multicenter prospective cohort study involving patients older than 75 years attending geriatric and nephrology outpatient services in participating institutions in Austria, Germany, Israel, Italy, the Netherlands, Poland and Spain. Only people aged 75 or more were asked to participate because of the high prevalence of CKD in this population [8, 27]. Methods of the SCOPE study have been extensively described elsewhere [24]. Briefly, all patients attending the outpatient services at participating centers from August 2016 to August 2018 were asked to participate. Only patients signing a written informed consent entered the 
study. Age greater or equal to 75 years was the only inclusion criteria, the exclusion criteria were: end-stage renal disease or dialysis at time of enrollment; history of solid organ or bone marrow transplantation; active malignancy within 24 months prior to screening or metastatic cancer; life expectancy less than 6 months (based on the judgment of the study physician after careful medical history collection and diagnoses emerging from examination of clinical documentation exhibited); severe cognitive impairment (Mini Mental State Examination < 10); any medical or other reason (e.g. known or suspected patients' inability to comply with the protocol procedure) in the judgement of the investigators, that the patient was unsuitable for the study; unwilling to provide consent and limited possibility to attend followup visits. Enrolled patients underwent an extensive assessment including: demographic data, socioeconomic status, physical examination, comprehensive geriatric assessment, bioimpedance analysis, diagnoses (clinical history and assessment of clinical documentation exhibited by patients and/or caregivers), quality of life, physical performance, overall comorbidity and blood and urine sampling. Patients were followed-up for 24-months as previously described [24]. The study protocol was approved by ethics committees at all participating institutions, and complies with the Declaration of Helsinki and Good Clinical Practice Guidelines. The study was registered at ClinicalTrials.gov (NCT02691546). Only baseline data was used in the present study.

Overall, 2461 patients were initially enrolled in the study, 310 participants were excluded because of missing data in any of the study variables, leaving a final sample of 2151 participants to be included in the analyses.

\section{Study variables}

Each participating center used standardized measurement methods in accordance with the study protocol. Age, gender and country were considered in the analysis.

\section{Anthropometric measurements}

Trained staff measured participants' height and weight using a weighing scale with height rod. Waist, hip, arm and calf circumference were measured with a flexible measuring tape to the nearest $0.1 \mathrm{~cm}$ according to the WHO's standard technique. Then, the Body Mass Index (BMI) was calculated by dividing the subject's weight in kilograms by height in metres squared, Waist-to-Height Ratio (WHtR) as WC divided by height and Waist-toHip Ratio (WHR) as quotient of the circumference of the waist to the hips.

\section{Risk of malnutrition (undernutrition)}

Nutritional status was assessed using the Mini Nutritional Assessment (MNA) questionnaire. This is simple and practical, non-invasive tool which is commonly used by health professionals for early detection of risk of malnutrition [28]. The MNA consists of 18 questions: 5 in the screening part and 13 in the assessment part. The total score ranges from 0 to 30 , where a score of less than 17 points indicates malnutrition, from 17 to 23.5 risk of malnutrition, and 24 or more points normal nutrition. The MNA is recommended by the ESPEN for detecting the presence of malnutrition and the risk of developing malnutrition among older adults [3].

\section{Biochemical markers of nutritional status}

Serum albumin was determined with the use of the bromocresol green colorimetric assay and presented as $\mathrm{g} / \mathrm{dl}$ [29]. Albumin is a serum hepatic protein with half-life of 14-20 days. Serum albumin level suggesting malnutrition was set at $<3.5 \mathrm{~g} / \mathrm{dL}$ (hypoalbuminemia) [30].

\section{Kidney function}

Kidney function was measured, according to estimated glomerular filtration rate (eGFR) category, based on the GFR BIS equation, which is among the few equations specifically developed and validated among people aged 70 or more $[31,32]$ :

$$
3736 \times \text { creatinine }^{-0.87} \times \text { age }^{-0.95}[\times 0.82 \text { if female }]
$$

Participants were divided into 4 groups presenting stages of CKD: G1-2 (60 or more); G3A (45-59.9); G3B (30-44.9); G4 (15-30), and were also dichotomized as $\mathrm{CKD}(-)$ group $(\mathrm{GFR} \geq 60)$ and $\mathrm{CKD}(+)$ group $(\mathrm{GRF}<60)$ [33].

\section{Overall comorbidity}

Overall comorbidity was assessed by Cumulative Illness Rating Score for Geriatrics (CIRS-G) [34]. CIRS-G consists of the assessment of the severity of coexisting diseases in 14 organ/systems scales, each ranging from 0 (problem absent) to 4 (severe problem with requirement of immediate treatment and/or severe organ/system failure).

\section{Diagnoses}

Diabetes, cancer, hypertension, coronary artery disease (CAD), cerebrovascular diseases (CVD), heart failure (HF), chronic obstructive pulmonary disease (COPD), and metabolic syndrome were also considered in the analyses. The occurrence of selected diagnoses was ascertained by the study physician through detailed collection of medical history and examination of clinical documentation exhibited by patients and caregivers 
during study visit as described above. Metabolic syndrome was defined on the basis of the International Diabetes Federation criteria as central obesity (based on WC) plus any two of the following risk factors: plasma triglycerides $\geq 150 \mathrm{mg} / \mathrm{dl}$, HDL-cholesterol< 40 for men and $<50$ for women, blood pressure $>130 / 80 \mathrm{mmHg}$ or previous diagnosis of hypertension, fasting plasma glucose $>100 \mathrm{mg} / \mathrm{dl}$ or previous diagnosis of diabetes [35].

\section{Statistical analysis}

All variables were checked for normality of distribution by the Kolmogorov-Smirnov test. All the continuous variables were not normally distributed, therefore they were presented by median and interquartile difference. Mann Whitney U-test was used to compare nutritional status between $\mathrm{CKD}(+)$ and $\mathrm{CKD}(-)$ groups and between men and women. Differences between subgroups, depending on CKD stages, were assessed using the Kruskal-Wallis and post hoc Dunn-Sidak test. Spearman correlations between nutritional status indicators and kidney function (eGFR) were calculated. Additionally, test of homogeneity of the slopes (comparison of regression lines and ANOVA interactions) were used to corroborate the different relationship of eGFR to nutritional components according to sex.

Logistic regression (Odds ratios and corresponding 95\% confidence intervals $(95 \% \mathrm{CI})$ ) was used to assess which independent variables predicted the presence of CKD (Model 1), malnutrition defined with MNA $<24$ (Model 2) and malnutrition defined with albumin $<3.5$ $\mathrm{g} / \mathrm{dL}$ (Model 3). Each model was adjusted for age and sex (A), age, sex, concomitant diseases (diabetes mellitus, cancer, HF, CAD, CVD, COPD, metabolic syndrome) and country (B).

Statistical significance was set at $p<0.05$. All statistical analyses were performed with SPSS version 24 (SPSS Inc., Chicago, IL, USA).

\section{Results}

We studied 2151 subjects (932 men) with median age $79.5 \pm 5.9$ years. A total of $1333(62 \%)$ participants had CKD (GRF $<60 \mathrm{ml} / \mathrm{min} / 1.73 \mathrm{~m}^{2}$ ). Table 1 shows the general characteristics of the participants divided by sex and presence of CKD.

Women showed lower educational level than men, had lower prevalence of diabetes mellitus, cancer, CAD, CVD, HF, COPD, and lower CIRS-G score. Men had higher weight, WHR, AC, WC, $\mathrm{HC}, \mathrm{CC}$ and higher MNA SF and MNA scores. Women were more frequent among patients enrolled in Germany and Poland, while people from Austria, Italy and the Netherlands were more frequently men (Table 1$)$.

Participants in the $\mathrm{CKD}(+)$ group were older, more frequently men, less educated and with a higher prevalence of chronic diseases, including metabolic syndrome. The nutritional status of subjects with CKD was characterized by higher BMI, WHR, WHtR, weight and $\mathrm{AC}, \mathrm{WC}, \mathrm{HC}$ and $\mathrm{CC}$. The result obtained in the MNA SF test did not differ between the groups, while the MNA and albumin were significantly lower in the $\mathrm{CKD}(+)$ group compared to patients without CKD. Finally, subjects with CKD were more frequent among patients enrolled in Austria and the Netherlands, while less frequent among those enrolled in Israel, Poland and Spain (Table 1).

Analysis of study variables across eGFR stages $1-4$ by sex (Table 2) showed that age increased and education decreased together with reducing eGFR. Higher values of anthropometric measures were observed among patients with more advanced CKD stages, while MNA and albumin level decreased together with reducing eGFR. Likewise, the prevalence of concomitant chronic diseases and CIRS-G continually increased together with eGFR stage. Finally, more advanced stages of CKD were especially prevalent among patients from Austria and the Netherlands, while less frequent in Israel, Italy and Poland (Table 2).

The correlations of eGFR with selected variables are presented in Table 3. Negative correlations with age and positive correlations with education level were found. Negative correlations for all the nutritional anthropometric variables (except for WHR - eGFR relationship in men) with kidney function were observed. A positive correlation occurred between eGFR and MNA score and albumin concentration (Table 3).

Figure 1a-d shows the relationship of eGFR to selected nutritional variables. Comparison of the slopes of regression lines showed significant differences for eGFR association to CC (Fig. 1a) and albumin levels (Fig. 1b) between men and women. In men, those associations were steeper than in women. For other nutritional variables, those associations were similar in both sexes. As an example, the relationship of eGFR to WHtR has been shown in Fig. 1c and that to MNA in Fig. 1d.

Age and sex adjusted regression models showed an increased risk of CKD (defined as eGFR <60) for higher weight, $\mathrm{AC}, \mathrm{WC}, \mathrm{HC}, \mathrm{CC}, \mathrm{BMI}$ and $\mathrm{WHtR}$, as well as a decrease in the risk of CKD with higher MNA and higher serum albumin. After further adjusting for comorbidities and country results remained substantially unchanged (Table 4).

Finally, a graded association between CKD stage and malnutrition defined with MNA $<24$ or hypoalbuminemia was found in age- and sex-adjusted model. After further adjustment for comorbidities and country, only eGFR $<30$ remained statistically associated with MNA < 24 or hypoalbuminemia (Table 4). 
Table 1 General characteristics of the study population $(n=2151)$ according to sex and prevalence of CKD. The quantitative values are expressed by median and interquartile difference, qualitative values as number and percentage

\begin{tabular}{|c|c|c|c|c|c|}
\hline Variable & $\begin{array}{l}\text { All } \\
\boldsymbol{n}=2151\end{array}$ & $\begin{array}{l}\text { Men } \\
\boldsymbol{n}=932\end{array}$ & $\begin{array}{l}\text { Women } \\
\boldsymbol{n}=1219\end{array}$ & $\begin{array}{l}\text { CKD(-) group } \\
\boldsymbol{n}=818\end{array}$ & $\begin{array}{l}\text { CKD }(+) \text { group } \\
\boldsymbol{n}=1333\end{array}$ \\
\hline Age (years) & $79.5(5.9)$ & $79.5(5.8)$ & $79.5(5.9)$ & $78.6(4.5)$ & $80.2(6.2)^{c}$ \\
\hline Men $(n, \%)$ & $932(43.3)$ & - & - & $315(38.5)$ & $617(46.3)^{c}$ \\
\hline Education (years) & $11(7)$ & $12(7)$ & $11(7)^{a}$ & $12(8)$ & $11(6)^{c}$ \\
\hline Weight (kg) & $72.0(17.5)$ & $78(15.6)$ & $67(16.3)^{c}$ & $70(18.4)$ & $74(18.9)^{c}$ \\
\hline Arm circumference $(\mathrm{cm})$ & $28(4.0)$ & $28(3.5)$ & $28(5.0)$ & $28(4.0)$ & $28(4.0)^{c}$ \\
\hline Waist circumference (cm) & $98(15)$ & $102(14)$ & $95(16)^{c}$ & $96(15)$ & $99(16)^{c}$ \\
\hline Hip circumference $(\mathrm{cm})$ & $104(11.0)$ & $104(9.0)$ & $104(14.0)^{\mathrm{a}}$ & $102(10.1)$ & $105(12.0)^{c}$ \\
\hline Calf circumference $(\mathrm{cm})$ & $36(5.0)$ & $37(5.0)$ & $35(5.0)^{c}$ & $36(5.0)$ & $36(6.0)^{c}$ \\
\hline $\mathrm{BMI}\left(\mathrm{kg} / \mathrm{m}^{2}\right)$ & $27.3(5.6)$ & $27.2(5.0)$ & $27.4(6.3)$ & $26.7(5.4)$ & $27.6(5.7)^{c}$ \\
\hline WHR & $0.93(0.11)$ & $0.98(0.09)$ & $0.89(0.09)^{c}$ & $0.92(0.12)$ & $0.93(0.11)^{c}$ \\
\hline WHtR & $0.60(0.10)$ & $0.60(0.09)$ & $0.60(0.10)$ & $0.59(0.10)$ & $0.61(0.09)^{c}$ \\
\hline MNA SF & $13(2)$ & $14(2)$ & $13(2)^{c}$ & $13(2)$ & $13(2)$ \\
\hline MNA & $27.0(3.0)$ & $27.0(2.5)$ & $26.5(3.0)^{c}$ & $27.0(2.5)$ & $26.5(3.0)^{c}$ \\
\hline Albumin (g/dL) & $4.22(0.5)$ & $4.30(0.5)$ & $4.20(0.4)$ & $4.30(0.4)$ & $4.20(0.4)^{c}$ \\
\hline Diabetes $(n, \%)$ & $532(24.7)$ & $279(29.9)$ & $253(20.8)^{c}$ & $154(18.8)$ & $378(28.4)^{c}$ \\
\hline Cancer $(n, \%)$ & $368(17.1)$ & $188(20.2)$ & $180(14.8)^{c}$ & $116(14.2)$ & $252(18.9)^{b}$ \\
\hline Hypertension (n,\%) & $1635(76.0)$ & 707 (75.9) & $928(76.1)$ & $542(66.3)$ & $1093(82.0)^{c}$ \\
\hline CAD $(n, \%)$ & $288(13.4)$ & $171(18.3)$ & $117(9.6)^{c}$ & $72(8.8)$ & $216(16.2)^{c}$ \\
\hline CVD (n,\%) & $268(12.5)$ & $132(14.2)$ & $136(11.2)^{c}$ & $87(10.6)$ & $181(13.6)^{a}$ \\
\hline$H F(n, \%)$ & $335(15.6)$ & $167(17.9)$ & $168(13.8)^{b}$ & $68(8.3)$ & $267(20.0)^{c}$ \\
\hline $\operatorname{COPD}(n, \%)$ & $252(11.7)$ & $156(16.7)$ & $96(7.9)^{c}$ & $70(8.6)$ & $182(13.7)^{b}$ \\
\hline Metabolic syndrome $(n, \%)$ & $1257(58.4)$ & $555(59.5)$ & $702(57.6)$ & $403(49.3)$ & $854(64.1)^{c}$ \\
\hline CIRS-G & $8(6)$ & $8(7)$ & $7(7)^{c}$ & $7(6)$ & $9(7)^{c}$ \\
\hline \multicolumn{6}{|l|}{ Country } \\
\hline Austria (n, \%) & $225(10.5)$ & $117(12.6)$ & $108(8.9)^{b}$ & $30(3.7)$ & $195(14.6)^{c}$ \\
\hline Germany (n, \%) & $270(12.6)$ & $83(8.9)$ & $187(15.3)^{c}$ & $106(13.0)$ & $164(12.3)$ \\
\hline Israel (n, \%) & $299(13.9)$ & $138(14.8)$ & $161(13.2)$ & $149(18.2)$ & $150(11.3)^{c}$ \\
\hline Italy (n, \%) & $436(20.3)$ & $221(23.7)$ & $215(17.6)^{c}$ & $155(18.9)$ & $281(21.1)$ \\
\hline The Netherlands (n, \%) & $285(13.2)$ & $160(17.2)$ & $125(10.3)^{c}$ & $90(11.0)$ & $195(14.6)^{a}$ \\
\hline Poland (n, \%) & $352(16.4)$ & $101(10.8)$ & $251(20.6)^{c}$ & $160(19.6)$ & $192(14.4)^{b}$ \\
\hline Spain $(n, \%)$ & $284(13.2)$ & $112(12.0)$ & $172(14.1)$ & $128(15.6)$ & $156(11.7)^{b}$ \\
\hline
\end{tabular}

\section{Discussion}

To the best of our knowledge, this is the first study to analyze the relationship between nutritional status and kidney function in community dwelling people aged 75+ not on dialysis and without ESRD. The results show a clear association between nutritional status and eGFR in this population. Findings about anthropometric measures generally point out that the severity of overweight and obesity is related to the prevalence of CKD. Albumin and MNA, but not MNA SF, correlate with kidney function and discriminate eGFR categories.
As other studies show [36], CKD is primarily associated with abdominal obesity. This type of obesity is characterized by an increase of adipose tissue surrounding the intra-abdominal organs. This phenotype is associated with metabolic disturbances and several chronic diseases [37, 38]. One of them is metabolic syndrome. There is growing evidence that metabolic syndrome is significantly associated with the risk of rapid eGFR decline $[39,40]$, and its high prevalence among people with CKD in our study suggests that this notion may also apply to older populations. The easiest method to assess 
Table 2 General characteristics of men $(n=931)$ and women $(n=1217)$ according to eGFR category. The quantitative values are expressed by median and interquartile difference, qualitative values as number and percentage

\begin{tabular}{|c|c|c|c|c|c|c|c|c|c|c|}
\hline \multirow[t]{2}{*}{ Variable } & \multicolumn{5}{|l|}{ Men } & \multicolumn{5}{|l|}{ Women } \\
\hline & $\begin{array}{l}\text { G1-2 } \\
(\geq 60) \\
\boldsymbol{N}=315\end{array}$ & $\begin{array}{l}\text { G3A } \\
(45-59.9) \\
\boldsymbol{N}=368\end{array}$ & $\begin{array}{l}\text { G3B } \\
(30-44.9) \\
\boldsymbol{N}=198\end{array}$ & $\begin{array}{l}\mathrm{G} 4 \\
(15-30) \\
\boldsymbol{N}=50\end{array}$ & $p$-value & $\begin{array}{l}\text { G1-2 } \\
(\geq 60) \\
\boldsymbol{N}=503\end{array}$ & $\begin{array}{l}\text { G3A } \\
(45-59.9) \\
\boldsymbol{N}=483\end{array}$ & $\begin{array}{l}\text { G3B } \\
(30-44.9) \\
\boldsymbol{N}=193\end{array}$ & $\begin{array}{l}\mathrm{G} 4 \\
(15-30) \\
\boldsymbol{N}=38\end{array}$ & $\boldsymbol{p}$-value \\
\hline Age (years) & $78.7(4.6)$ & $79.6(6.0)$ & $80.1(6.0)$ & $83.2(7.3)$ & $<0.001^{\text {abcdef }}$ & $78.6(4.4)$ & $79.7(5.7)$ & $83.1(7.9)$ & $81.9(6.8)$ & $<0.001$ abcdef \\
\hline Education (years) & $12(8)$ & $12(7)$ & $11(6)$ & $9(5)$ & 0.031 bcdef & $12(7)$ & $11(6)$ & $10(5)$ & $8(3)$ & $<0.001$ abcdef \\
\hline Weight (kg) & $77.0(14.3)$ & $78.0(17.0)$ & $81.4(16.2)$ & $75.5(18.0)$ & $<0.001$ abcdef & $65.0(16.0)$ & $67.0(18.0)$ & $69.4(19.6)$ & $72.7(16.6)$ & $<0.001$ abcdef \\
\hline $\begin{array}{l}\text { Arm circumference } \\
(\mathrm{cm})\end{array}$ & $28(3.8)$ & $28(3.0)$ & $29(3.0)$ & $28(5.0)$ & $<0.001^{\text {bdf }}$ & $28(4.5)$ & $28(4.5)$ & $28(5.0)$ & $28(5.0)$ & $<0.001$ bcde \\
\hline $\begin{array}{l}\text { Waist circumference } \\
\text { (cm) }\end{array}$ & $100(14.0)$ & $101(13.9)$ & $104(13.0)$ & $102(14.8)$ & $<0.001^{\text {bdf }}$ & $92(17.0)$ & $95(16.0)$ & $98(16.0)$ & $102(13.2)$ & $<0.001$ abcdef \\
\hline $\begin{array}{l}\text { Hip circumference } \\
(\mathrm{cm})\end{array}$ & $102(9.0)$ & $103(9.0)$ & $106(9.0)$ & $105(10.0)$ & $<0.001$ bcde & $102(12.0)$ & $105(13.4)$ & $108(15.0)$ & $111(11.0)$ & $<0.001$ abcdef \\
\hline $\begin{array}{l}\text { Calf circumference } \\
\text { (cm) }\end{array}$ & $36(4)$ & $36(5)$ & $38(7)$ & $42(8)$ & $<0.001^{\text {bcdef }}$ & $35(4)$ & $36(5)$ & $36(6)$ & $41(13)$ & $<0.001$ abcef \\
\hline BMI $\left(\mathrm{kg} / \mathrm{m}^{2}\right)$ & $26.9(4.5)$ & $27.2(5.0)$ & $27.7(4.9)$ & $26.7(4.7)$ & 0.011 abcef & $26.6(5.8)$ & $27.6(6.0)$ & $28.5(7.0)$ & $29.6(7.5)$ & $<0.001$ abcdef \\
\hline WHR & $1(0.1)$ & $1(0.1)$ & $1(0.1)$ & $1(0.1)$ & ns & $0.89(0.1)$ & $0.89(0.1)$ & $0.89(0.1)$ & $0.90(0.1)$ & ns \\
\hline WHtR & $0.59(0.1)$ & $0.60(0.1)$ & $0.61(0.1)$ & $0.60(0.1)$ & $<0.001$ bcdef & $0.6(0.1)$ & $0.6(0.1)$ & $0.6(0.1)$ & $0.7(0.1)$ & $<0.001$ cef \\
\hline MNA SF & $14(2)$ & $14(1)$ & $14(2)$ & $14(3)$ & ns & $13(2)$ & $13(2)$ & $13(2)$ & $13(3)$ & ns \\
\hline MNA & $27(2.5)$ & $27(2.5)$ & $27(2.5)$ & $26(4.7)$ & $<0.001$ cef & $27(3.0)$ & $26.5(3.5)$ & $26(3.0)$ & $25.0(6.2)$ & $<0.001^{c}$ \\
\hline Albumin $(\mathrm{g} / \mathrm{dL})$ & $4.3(0.4)$ & $4.3(0.4)$ & $4.3(0.4)$ & $4.0(0.5)$ & $<0.001^{\text {cef }}$ & $4.3(0.4)$ & $4.2(0.5)$ & $4.2(0.5)$ & $4.0(0.6)$ & $<0.001^{c}$ \\
\hline Diabetes (n, \%) & $75(23.8)$ & $104(28.3)$ & $83(41.9)$ & $17(34.0)$ & $<0.001$ abcdef & $79(15.7)$ & $88(18.2)$ & $70(36.3)$ & $16(42.1)$ & $<0.001$ abcdef \\
\hline Cancer (n, \%) & $51(16.2)$ & $76(20.7)$ & $49(24.7)$ & $12(24.0)$ & ns & $65(12.9)$ & $78(16.1)$ & $29(15.0)$ & $6(15.8)$ & ns \\
\hline Hypertension (n, \%) & 201 (63.8) & $281(76.4)$ & $177(89.4)$ & $47(94.0)$ & $<0.001$ abcdef & $341(67.8)$ & 378 (78.3) & 169 (87.6) & $38(100.0)$ & $<0.001$ abcdef \\
\hline CAD (n, \%) & $36(21.2)$ & $68(40.0)$ & $56(32.9)$ & $10(5.9)$ & $<0.001$ abcdef & $36(7.2)$ & $36(7.5)$ & $36(18.7)$ & $9(23.7)$ & $<0.001$ bcdef \\
\hline CVD (n, \%) & $39(12.4)$ & $57(15.5)$ & $30(15.2)$ & $6(12.0)$ & ns & $48(9.5)$ & $49(10.1)$ & $30(15.5)$ & $9(23.7)$ & 0.009 bcdef \\
\hline $\mathrm{CHF}(\mathrm{n}, \%)$ & $26(8.3)$ & $74(20.1)$ & $51(25.8)$ & $15(30.0)$ & $<0.001$ abcdef & $42(8.3)$ & $75(15.5)$ & $40(20.7)$ & $11(28.9)$ & $<0.001$ abcdef \\
\hline COPD (n, \%) & $40(12.7)$ & $59(16.0)$ & $44(22.2)$ & $13(26.0)$ & 0.011 abcdef & $30(6.0)$ & $36(7.5)$ & $25(13.0)$ & $5(13.2)$ & $0.012^{\text {abcdef }}$ \\
\hline $\begin{array}{l}\text { Metabolic syndrome } \\
(\mathrm{n}, \%)\end{array}$ & $161(51.1)$ & $213(57.9)$ & $145(73.2)$ & $36(72.0)$ & $<0.001$ abcdef & $242(48.1)$ & $289(59.8)$ & $140(72.5)$ & $30(78.9)$ & $<0.001$ abcdef \\
\hline CIRS-G & $7(6)$ & $8(6)$ & $10(8)$ & $12(7)$ & $<0.001$ abcdef & $6(5)$ & $7(6)$ & $11(7)$ & $12(6)$ & $<0.001$ abcde \\
\hline \multicolumn{11}{|l|}{ Country } \\
\hline Austria (n, \%) & $12(3.8)$ & $34(9.2)$ & $44(22.2)$ & $26(52.0)$ & $<0.001$ abcdef & $18(3.6)$ & $37(7.7)$ & $34(17.6)$ & $18(47.4)$ & $<0.001$ abcdef \\
\hline Germany (n, \%) & $32(10.2)$ & $35(9.5)$ & $12(6.1)$ & $4(8.0)$ & ns & $74(14.7)$ & $69(14.3)$ & $39(20.2)$ & $5(13.2)$ & ns \\
\hline Israel (n, \%) & $64(20.3)$ & $54(14.7)$ & $17(8.6)$ & $3(6.0)$ & 0.001 abcde & $85(16.9)$ & $58(12.0)$ & $16(8.3)$ & $1(2.6)$ & $0.002^{\text {abcdef }}$ \\
\hline Italy (n, \%) & $73(23.2)$ & $101(27.4)$ & $44(22.2)$ & $3(6.0)$ & $0.008^{\text {acdef }}$ & $82(16.3)$ & $107(22.2)$ & $23(11.9)$ & $3(7.9)$ & $0.003^{\text {abcdef }}$ \\
\hline $\begin{array}{l}\text { The Netherlands } \\
(n, \%)\end{array}$ & $43(13.7)$ & $61(16.6)$ & $46(23.2)$ & $10(20.0)$ & $0.042^{a b c d e f}$ & $47(9.3)$ & $40(8.3)$ & $32(16.6)$ & $6(15.8)$ & $0.008^{\text {cde }}$ \\
\hline Poland (n, \%) & $49(15.6)$ & $42(11.4)$ & $10(5.1)$ & $0(0.0)$ & $<0.001$ abcdef & $111(22.1)$ & $115(23.8)$ & $25(13.0)$ & $0(0.0)$ & $<0.001$ bcdef \\
\hline Spain $(n, \%)$ & $42(13.3)$ & $41(11.1)$ & $25(12.6)$ & $4(8.0)$ & ns & $86(17.1)$ & $57(11.8)$ & $24(12.4)$ & $5(13.2)$ & ns \\
\hline
\end{tabular}

${ }^{*} p$-values $=$ based on comparisons between subgroups

${ }^{a} \mathrm{G} 1-2$ (60 or more) vs G3A (45-59.9)

b $\mathrm{G} 1-2$ (60 or more) vs G3B (30-44.9)

' $\mathrm{G} 1-2$ (60 or more) vs G4 (15-30)

${ }^{d} \mathrm{G} 3 \mathrm{~A}(45-59.9)$ vs G3B (30-44.9)

${ }^{e} \mathrm{G} 3 \mathrm{~A}(45-59.9)$ vs G4 (15-30)

${ }^{f} \mathrm{G} 3 \mathrm{~B}(30-44.9)$ vs G4 (15-30)

the occurrence of this type of obesity is the WC [41]. Central obesity was defined based on cut-off points advocated by Lean et al. (1995) as a WC of $\geq 102 \mathrm{~cm}$ in men, and $\geq 88 \mathrm{~cm}$ in women [42]. Such WC are one of the risk factors of metabolic syndrome, hypertension, diabetes, which indirectly affects kidney function. In our 
Table 3 Spearmans correlations between nutritional status indicators and eGFR

\begin{tabular}{|c|c|c|c|}
\hline Variable & $\begin{array}{l}\text { Correlation with GFR } \\
\text { All }\end{array}$ & $\begin{array}{l}\text { Correlation with GFR } \\
\text { Men }\end{array}$ & $\begin{array}{l}\text { Correlation with GFR } \\
\text { Women }\end{array}$ \\
\hline Age (years) & $-0.274^{b}$ & $-0.257^{b}$ & $-0.286^{b}$ \\
\hline Education (years) & $0.106^{b}$ & $0.066^{b}$ & $0.150^{b}$ \\
\hline Weight (kg) & $-0.164^{b}$ & $-0.098^{b}$ & $-0.152^{b}$ \\
\hline Arm circumference $(\mathrm{cm})$ & $-0.096^{b}$ & $-0.113^{b}$ & $-0.086^{b}$ \\
\hline Waist circumference $(\mathrm{cm})$ & $-0.186^{b}$ & $-0.150^{b}$ & $-0.160^{b}$ \\
\hline Hip circumference (cm) & $-0.168^{b}$ & $-0.154^{b}$ & $-0.189^{b}$ \\
\hline Calf circumference $(\mathrm{cm})$ & $-0.167^{b}$ & $-0.188^{b}$ & $-0.131^{b}$ \\
\hline $\mathrm{BMI}\left(\mathrm{kg} / \mathrm{m}^{2}\right)$ & $-0.118^{b}$ & $-0.073^{a}$ & $-0.153^{b}$ \\
\hline WHR & $-0.100^{b}$ & -0.053 & $-0.045^{a}$ \\
\hline $\mathrm{WH} \mathrm{tR}$ & $-0.135^{b}$ & $-0.122^{b}$ & $-0.145^{b}$ \\
\hline MNA SF & 0.018 & 0.023 & 0.031 \\
\hline MNA & $0.111^{b}$ & $0.102^{b}$ & $0.134^{b}$ \\
\hline Albumin (g/dL) & $0.092^{b}$ & $0.103^{b}$ & $0.094^{b}$ \\
\hline
\end{tabular}

${ }^{\mathrm{a}} p<0.05 ;{ }^{\mathrm{b}} p<0.01$

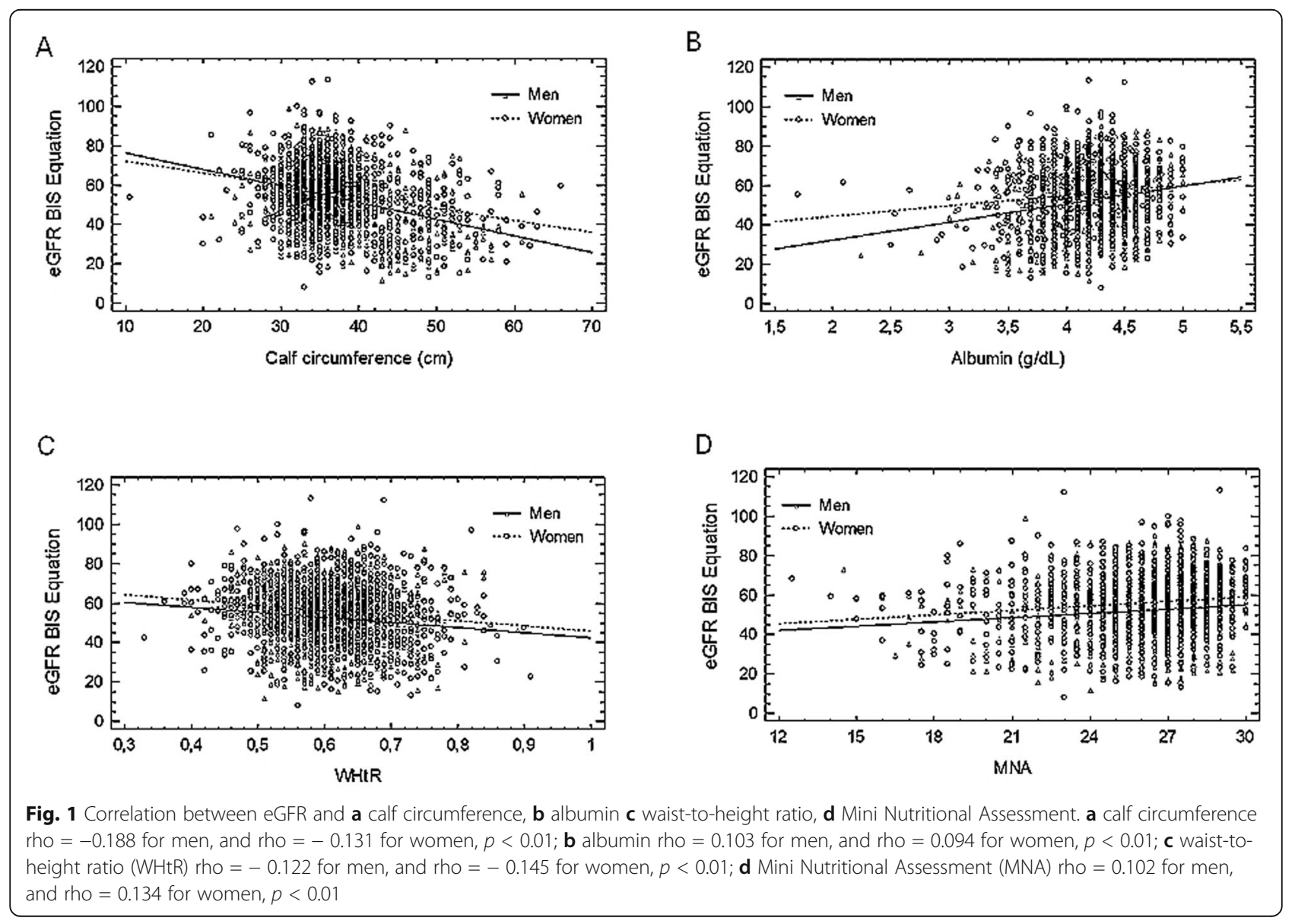


Table 4 Logistic regression models (Odds ratios and corresponding 95\% confidence intervals (95\%Cl)) for the association of CKD and malnutrition: the presence of CKD (Model 1) predicted by nutritional and anthropometric variables, while malnutrition defined with MNA < 24 (Model 2) and malnutrition defined with albumin < $3.5 \mathrm{~g} / \mathrm{dL}$ (Model 3) predicted by CKD stages. Each model was adjusted for age and sex (A), age, sex, concomitant diseases (diabetes mellitus, cancer, HF, CAD, CVD, COPD, metabolic syndrome) and country (B)

\begin{tabular}{|c|c|c|c|c|}
\hline \multirow[t]{2}{*}{ Variables } & \multicolumn{2}{|l|}{$\begin{array}{l}\text { Model 1A } \\
\text { CKD } \\
\text { GRF }<60 \mathrm{ml} / \mathrm{min} / 1.73 \mathrm{~m}^{2}\end{array}$} & \multicolumn{2}{|c|}{$\begin{array}{l}\text { Model 1B } \\
\text { CKD } \\
\text { GRF }<60 \mathrm{ml} / \mathrm{min} / 1.73 \mathrm{~m}^{2}\end{array}$} \\
\hline & \multicolumn{2}{|l|}{$\begin{array}{l}\text { OR }(95 \% \mathrm{Cl}) \\
\text { age and sex adjusted }\end{array}$} & \multicolumn{2}{|c|}{$\begin{array}{l}\text { OR }(95 \% \mathrm{Cl}) \\
\text { age, sex, comorbidities }{ }^{\mathrm{a}} \text { and country adjusted }\end{array}$} \\
\hline Weight (kg) & \multicolumn{2}{|l|}{$1.03(1.02-1.04)$} & \multicolumn{2}{|l|}{$1.02(1.01-1.03)$} \\
\hline Arm Circumference (cm) & \multicolumn{2}{|l|}{$1.10(1.07-1.13)$} & \multicolumn{2}{|l|}{$1.07(1.04-1.10)$} \\
\hline Waist circumference (cm) & \multicolumn{2}{|l|}{$1.03(1.02-1.03)$} & \multicolumn{2}{|l|}{$1.01(1.00-1.02)$} \\
\hline Hip circumference (cm) & \multicolumn{2}{|l|}{$1.03(1.02-1.04)$} & \multicolumn{2}{|l|}{$1.02(1.01-1.03)$} \\
\hline Calf circumference $(\mathrm{cm})$ & \multicolumn{2}{|l|}{$1.08(1.06-1.10)$} & \multicolumn{2}{|l|}{$1.03(1.00-1.06)$} \\
\hline BMI $\left(\mathrm{kg} / \mathrm{m}^{2}\right)$ & \multicolumn{2}{|l|}{$1.07(1.05-1.09)$} & \multicolumn{2}{|l|}{$1.05(1.02-1.07)$} \\
\hline WHR & \multicolumn{2}{|l|}{$1.10(0.97-1.25)$} & \multicolumn{2}{|l|}{$0.99(0.86-1.14)$} \\
\hline WHtR & \multicolumn{2}{|l|}{$1.38(1.22-1.56)$} & \multicolumn{2}{|l|}{$1.18(1.01-1.38)$} \\
\hline MNA & \multicolumn{2}{|l|}{$0.95(0.92-0.99)$} & \multicolumn{2}{|l|}{$0.98(0.94-1.00)$} \\
\hline Albumin (g/dL) & \multicolumn{2}{|l|}{$0.73(0.56-0.95)$} & \multicolumn{2}{|l|}{$0.73(0.53-1.00)$} \\
\hline \multirow[t]{2}{*}{$\begin{array}{l}\text { Variables } \\
\text { CKD stages } \\
\text { GFR in } \mathrm{ml} / \mathrm{min} / 1.73 \mathrm{~m}^{2}\end{array}$} & $\begin{array}{l}\text { Model } 2 \mathbf{A} \\
\text { malnutrition } \\
\text { MNA }<24\end{array}$ & $\begin{array}{l}\text { Model 2B } \\
\text { malnutrition } \\
\text { MNA }<24\end{array}$ & $\begin{array}{l}\text { Model } \mathbf{3 A} \\
\text { malnutrition } \\
\text { albumin }<3.5\end{array}$ & $\begin{array}{l}\text { Model 3B } \\
\text { malnutrition } \\
\text { albumin }<3.5\end{array}$ \\
\hline & $\begin{array}{l}\text { OR }(95 \% \mathrm{Cl}) \\
\text { age and sex adjusted }\end{array}$ & $\begin{array}{l}\text { OR }(95 \% \mathrm{Cl}) \\
\text { age, sex, comorbidities }{ }^{\mathrm{a}} \text { and } \\
\text { country adjusted }\end{array}$ & $\begin{array}{l}\text { OR }(95 \% \mathrm{Cl}) \\
\text { age and sex adjusted }\end{array}$ & $\begin{array}{l}\text { OR }(95 \% \mathrm{Cl}) \\
\text { age, sex, comorbidities }^{\mathrm{a}} \\
\text { and country adjusted }\end{array}$ \\
\hline$C K D \geq 60$ & 1 & 1 & 1 & 1 \\
\hline CKD 45-59.9 & $1.12(0.86-1.45)$ & $1.03(0.78-1.37)$ & $2.05(1.02-4.10)$ & $1.70(0.78-3.72)$ \\
\hline CKD 30-44.9 & $1.51(1.14-2.00)$ & $1.28(0.93-1.75)$ & $2.21(1.20-4.06)$ & $1.71(0.81-3.61)$ \\
\hline CKD 15-30 & $3.15(1.98-4.99)$ & $2.95(1.77-4.94)$ & $3.70(1.57-8.67)$ & $5.54(1.66-18.51)$ \\
\hline
\end{tabular}

${ }^{a}$ comorbidities: diabetes, cancer, HF, CAD, CVD, COPD, metabolic syndrome

study, WC was strongly associated with CKD. Similarly to the study of Evans et al., where researchers emphasize that abdominal obesity is even more strongly associated with CKD risk than BMI [43]. Although BMI is a controversial indicator and there is ongoing discussion regarding the cut-off points for the older adults [44], present analysis showed that the occurrence of kidney disease is associated with higher BMI.

WHtR was another indicator that showed a link between CKD and obesity. This indicator is a universal measure that can be used regardless of sex and race with a cut-off value of $\mathrm{WHtR}=0.5$. The median for the CKD $(+)$ group was high $(0.6 \pm 0.1)$, and reached the highest value of $0.7 \pm 0.1$ in the group of women in CKD stage G4. In the meta-analyses done by Lee et al. [45] and Correa et al. [46], WHtR was the best discriminator for the risk of hypertension, diabetes, and dyslipidemia in both sexes. WHtR can be simpler and more predictive indicator of the cardiometabolic risk factors associated with central obesity than other anthropometric indices $[47,48]$. In the present study WHtR showed the highest odds ratio for the prediction of CKD. The increase of WHtR by 0.1 increased the risk of kidney disease by $38 \%$, and by $18 \%$ after adjustment for comorbidities. This easy-to-count indicator can be recommended as a CKD risk screening tool.

In contrast, WHR, as a body build indicator can only be analysed separately for sexes and only for participants categorized as obese in order to assess obesity type $[49,50]$. The lack of statistically significant correlation between eGFR and WHR in men and lack of statistical significance in logistic regression analyses seems to suggest minor relevance of this measure in older population.

Non-dominant middle arm circumference constitutes a useful tool as a marker of malnutrition [51]. Our analysis showed a negative correlation with eGFR but it presented a small differentiation between eGFR categories (about $1 \mathrm{~cm}$ ). Additionally, AC may be loaded with one of the highest anthropometric measurement errors [52]. Therefore, inference based on this result should be limited in older population. 
In contrast, the CC showed higher differentiation between eGFR categories. Differences found between subgroups according to the eGFR category, the highest statistically significant correlation coefficients with eGFR and statistical significance in logistic regression analyses indicate a strong relationship between $\mathrm{CC}$ and kidney function.

Men with eGFR 15-30 had lower BMI, WHtR and WC as compared to the group of men with eGFR 3044.9, while the $C C$ was definitely higher. This may be due to the presence of oedema of the lower limbs that accompanies advanced CKD. The lowest values of albumin observed in this group may also partly account for this apparent discrepancy [53, 54]. In women, albumin levels also decrease what is accompanied by an increase in $\mathrm{CC}$ but at the same time weight, BMI and WHtR were also higher. A detailed analysis of body composition should clarify those potential sex differences.

Albumin level and MNA were used as markers of nutritional status and malnutrition. Decrease in albumin level may be associated with a decrease in renal function and loss of albumin in the urine. This phenomenon is escalating with the progression of CKD [55]. Regression analysis suggests that irrespective of confounders, an increase in albumin of $1 \mathrm{~g} / \mathrm{dL}$ reduces the likelihood of CKD by $27 \%$. Likewise, advanced CKD (eGFR $<30$ ) increases the likelihood of malnutrition (albumin $<3,5 \mathrm{~g}$ / $\mathrm{dL}$ ) more than three times (five times after adjusting for comorbidities and country). This data confirms the strong relationship between eGFR and albumin levels.

One of the most important findings was the poorer MNA tests results in CKD participants. Generally, the studied group of older people was relatively fit and with good nutritional status. According to MNA score $<24$ a risk of malnutrition was observed in only 338 subjects (15.7\%). Nevertheless, this risk increased together with reduced kidney function. Positive correlation for both genders and also statistically lower MNA result in the group with eGFR 15-30 indicates an increasing risk of malnutrition in CKD subjects. In contrast, the MNA SF screening test did not significantly correlate with eGFR nor differ between eGFR stages, which may suggest limited application of MNA SF as malnutrition screening tool in older adults $75+$ [56].

Overall, findings from the present study may be relevant to clinicians dealing with older patients. The assessment of anthropometric measures and comorbidities may help to identify patients at risk of negative cardiometabolic and renal outcomes to be referred to specific care pathways aimed at reducing the burden of abdominal obesity and other risk factors. On the other hand, the identification of patients at risk of malnutrition is equally relevant when considering the consequences of malnutrition in terms of frailty, functional decline, sarcopenia, falls, hospitalization and death. In both cases, regular and careful monitoring of nutritional status using validated instruments would be needed.

Limitations of our study deserve to be mentioned. The cross-sectional design limits the interpretation of the observed associations, and the analysis of currently ongoing prospective phase of the SCOPE study may reveal different results. The population in the initial study included people with no advanced chronic kidney disease, and therefore inferences may relate to risk factors rather than symptoms in the final stages of CKD. We did not analyze the impact of diet on our findings. The observation that excessive body weight, and especially visceral obesity is associated with CKD, whereas malnutrition may appear in advanced stage of chronic kidney disease in both sexes suggests the need for a personalized diet and appropriately selected physical activity to prevent both malnutrition and obesity [3]. However, 24 h-dietary recall data will be available within the SCOPE study and warrants further analyses in this very interesting topic. Body composition, as well as steroid hormones and hypothalamic-pituitary-adrenal/gonadix axis were not included in the present study. Nevertheless, both are planned to be included in future studies within the SCOPE project.

As for strength, we studied a real world population of older people aged 75 or more enrolled without stringent exclusion criteria who underwent a comprehensive assessment of anthropometric and nutritional variables measured by standardized methods.

\section{Conclusions}

The population of community dwelling $75+$ with CKD shows generally features of overweight and obesity with small prevalence of malnutrition. For anthropometric measures, the strongest association with eGFR and the highest odds of CKD were identified using WC, HC, AC, CC and WHtR. Albumin level and MNA, but not MNA SF, indicated an increasing odds of malnutrition together with decreasing eGRF.

\section{Abbreviations \\ AC: Middle arm circumference; BMI: Body mass index; CAD: Coronary artery disease; CC: Calf circumference; CIRS-G: Cumulative Illness Rating Score for Geriatrics; CKD: Chronic kidney disease; COPD: Chronic obstructive pulmonary disease; CVD: Cerebro Vascular Disease; eGFR: Estimated glomerular filtration rate; ESRD: End stage renal disease; HC: Hip circumference; HF: Heart failure; MNA SF: Short Form of Mini Nutrition Assessment; MNA: Mini Nutrition Assessment; SCOPE: Screening for Chronic Kidney Disease Among Older People Across Europe; WC: Waist circumference; WHR: Waist-to-Hip ratio; WHtR: Waist-to-Height ratio}

\section{Acknowledgements}

SCOPE study investigators

Coordinating center, Fabrizia Lattanzio, Italian National Research Center on Aging (INRCA), Ancona, Italy - Principal Investigator. Andrea Corsonello, Silvia Bustacchini, Silvia Bolognini, Paola D'Ascoli, Raffaella Moresi, Giuseppina Di Stefano, Cinzia Giammarchi, Anna Rita Bonfigli, Roberta Galeazzi, Federica 
Lenci, Stefano Della Bella, Enrico Bordoni, Mauro Provinciali, Robertina Giacconi, Cinzia Giuli, Demetrio Postacchini, Sabrina Garasto, Annalisa Cozza, Francesco Guarasci, Sonia D'Alia - Italian National Research Center on Aging (INRCA), Ancona, Fermo and Cosenza, Italy - Coordinating staff. Romano Firmani, Moreno Nacciariti, Mirko Di Rosa, Paolo Fabbietti - Technical and statistical support.

Participating centers

- Department of Internal Medicine, Medical University of Graz, Austria: Gerhard Hubert Wirnsberger, Regina Elisabeth Roller-Wirnsberger, Carolin Herzog, Sonja Lindner

- Section of Geriatric Medicine, Department of Internal Medicine, Erasmus MC, University Medical Center Rotterdam, The Netherlands: Francesco Mattace-Raso, Lisanne Tap, Gijsbertus Ziere, Jeannette Goudzwaard. - Department of Geriatrics, Healthy Ageing Research Centre, Medical University of Lodz, Poland: Tomasz Kostka, Agnieszka Guligowska, Łukasz Kroc, Bartłomiej K Sołtysik, Małgorzata Pigłowska, Agnieszka Wójcik, Zuzanna Chrząstek, Natalia Sosowska, Anna Telążka, Joanna Kostka, Elizaveta Fife, Katarzyna Smyj, Kinga Zel.

- The Recanati School for Community Health Professions at the faculty of Health Sciences at Ben-Gurion University of the Negev, Israel: Rada ArtziMedvedik, Yehudit Melzer, Mark Clarfield, Itshak Melzer; and Maccabi Healthcare services southern region, Israel: Rada Artzi-Medvedik, Ilan Yehoshua, Yehudit Melzer.

- Geriatric Unit, Internal Medicine Department and Nephrology Department, Hospital Universitari de Bellvitge, Institut d'Investigació Biomèdica de Bellvitge - IDIBELL, L'Hospitalet de Llobregat, Barcelona, Spain: Francesc Formiga, Rafael Moreno-González, Xavier Corbella, Yurema Martínez, Carolina Polo, Josep Maria Cruzado.

- Department of Geriatric Medicine, Hospital Clínico San Carlos, Madrid: Pedro Gil Gregorio, Sara Laínez Martínez, Mónica González Alonso, Jose A Herrero Calvo, Fernando Tornero Molina, Lara Guardado Fuentes, Pamela Carrillo García, María Mombiedro Pérez.

- Department of General Internal Medicine and Geriatrics, Krankenhaus Barmherzige Brüder Regensburg and Institute for Biomedicine of Aging, Friedrich-Alexander-Universität Erlangen-Nürnberg, Germany: Alexandra Renz, Susanne Muck, Stephan Theobaldy, Andreas Bekmann, Revekka Kaltsa, Sabine Britting, Robert Kob, Christian Weingart, Ellen Freiberger, Cornel Sieber. - Department of Medical Sciences, Uppsala University, Sweden: Johan Ärnlöv, Axel Carlsson, Tobias Feldreich.

We thank the BioGer IRCCS INRCA Biobank for the collection of the SCOPE samples.

Scientific advisory board (SAB)

Roberto Bernabei, Catholic University of Sacred Heart, Rome, Italy Christophe Bula, University of Lausanne, Switzerland

Hermann Haller, Hannover Medical School, Hannover, Germany Carmine Zoccali, CNR-IBIM Clinical Epidemiology and Pathophysiology of Renal Diseases and Hypertension, Reggio Calabria, Italy Data and Ethics Management Board (DEMB)

Dr. Kitty Jager, University of Amsterdam, The Netherlands

Dr. Wim Van Biesen, University Hospital of Ghent, Belgium

Paul E. Stevens, East Kent Hospitals University NHS Foundation Trust, Canterbury, United Kingdom

We thank the BioGer IRCCS INRCA Biobank for the collection of the SCOPE samples.

\section{About this supplement}

This article has been published as part of BMC Geriatrics Volume 20 Supplement 1 2020: The Screening for Chronic Kidney Disease among Older People across Europe (SCOPE) project: findings from cross-sectional analysis. The full contents of the supplement are available at https://bmcgeriatr.biomedcentral.com/articles/supplements/volume-20-supplement-1.

\section{Authors' contributions}

AG, MP and TK participated in manuscript drafting and revising, participated in study protocol design and data collection. AC and FL conceived the study, coordinated study protocol and data collection, participated in manuscript drafting and revising. PF participated in statistical analyses, manuscript drafting and revising. RRW participated in study protocol design, data collection, writing of the manuscript and taking responsibility for the publication process. GW, FMR, LT, PG, SLM, IM, RAM, IY, FF, RMG, CS, CW, JÄ,
ACC participated in study protocol design, data collection, and manuscript revision and approval. All authors read and approved the final manuscript.

\section{Funding}

SCOPE study and publication costs are funded by the European Union Horizon 2020 program, under the Grant Agreement n 634869. Funding body had no role in the design of the study and collection, analysis, and interpretation of data and in writing the manuscript.

\section{Availability of data and materials}

Data will be available for SCOPE researchers through the project website (www.scopeproject.eu).

\section{Ethics approval and consent to participate}

The study protocol was approved by ethics committees at all participating institutions, and complies with the Declaration of Helsinki and Good Clinical Practice Guidelines. All patients signed a written informed consent to be enrolled. Only baseline data are used in the present study. Ethics approvals have been obtained by Ethics Committees in participating institutions as follows:

Italian National Research Center on Aging (INRCA), Italy, \#2015 0522 IN, January 27, 2016.

University of Lodz, Poland, \#RNN/314/15/KE, November 17, 2015. Medizinische Universität Graz, Austria, \#28-314 ex 15/16, August 5, 2016 Erasmus Medical Center Rotterdam, The Netherlands, \#MEC-2016-036 \#NL56039.078.15, v.4, March 7, 2016.

Hospital Clínico San Carlos, Madrid, Spain, \# 15/532-E_BC, September 16, 2016

Bellvitge University Hospital Barcellona, Spain, \#PR204/15, January 29, 2016. Friedrich-Alexander University Erlangen-Nürnberg, Germany, \#340_15B, January 21, 2016.

Helsinki committee in Maccabi Healthcare services, Bait Ba-lev, Bat Yam, Israel, \#45/2016, July 24, 2016.

\section{Consent for publication}

Not applicable.

\section{Competing interests}

The authors declare that they have no competing interests.

\section{Author details}

${ }^{1}$ Department of Geriatrics, Healthy Ageing Research Centre, Medical University of Lodz, Łódź, Poland. ${ }^{2}$ Italian National Research Center on Aging (IRCCS INRCA), Ancona, Fermo and Cosenza, Italy. ${ }^{3}$ Laboratory of Geriatric Pharmacoepidemiology and Biostatistics, IRCCS INRCA, Via S. Margherita 5, 60124 Ancona, Italy. ${ }^{4}$ Department of Internal Medicine, Medical University of Graz, Graz, Austria. ${ }^{5}$ Department of Medical Sciences, Uppsala University, Uppsala, Sweden. ${ }^{6}$ School of Health and Social Studies, Dalarna University, Falun, Sweden. ${ }^{7}$ Division of Family Medicine, Department of Neurobiology, Care Sciences and Society, Karolinska Institutet, Huddinge, Sweden. ${ }^{8}$ Section of Geriatric Medicine, Department of Internal Medicine, Erasmus MC, University Medical Center Rotterdam, Rotterdam, The Netherlands. ${ }^{9}$ Geriatric Unit, Internal Medicine Department, Bellvitge University Hospital - IDIBELL L'Hospitalet de Llobregat, Barcelona, Spain. ${ }^{10}$ Department of Internal Medicine-Geriatrics, Institute for Biomedicine of Aging, Krankenhaus Barmherzige Brüder, Friedrich-Alexander Universität Erlangen-Nürnberg, Koberger Strasse 60, 90408 Nuremberg, Germany. ${ }^{11}$ Geriatric Department, Hospital Clínico San Carlos, Madrid, Spain. ${ }^{12}$ The Recanati School for Community Health Professions at the Faculty of Health Sciences at Ben-Gurion University of the Negev, Beer-sheva, Israel. ${ }^{13}$ Maccabi Healthcare Services Southern Region, Tel Aviv-Yafo, Israel.

Received: 5 August 2020 Accepted: 11 August 2020 Published: 2 October 2020

\section{References}

1. GBD Chronic Kidney Disease Collaboration. Global, regional, and national burden of chronic kidney disease, 1990-2017: a systematic analysis for the global burden of disease study 2017. Lancet. 2020;395(10225):709-33. 
2. Hill NR, Fatoba ST, Oke JL, Hirst JA, O'Callaghan CA, Lasserson DS, Hobbs FD. Global prevalence of chronic kidney disease - a systematic review and meta-analysis. PLoS One. 2016;11(7):e0158765.

3. Volkert D, Beck AM, Cederholm T, Cruz-Jentoft A, Goisser S, Hooper L, Kiesswetter E, Maggio M, Raynaud-Simon A, Sieber CC, et al. ESPEN guideline on clinical nutrition and hydration in geriatrics. Clin Nutr. 2019; 38(1):10-47.

4. Gallus S, Lugo A, Murisic B, Bosetti C, Boffetta P, La Vecchia C. Overweight and obesity in 16 European countries. Eur J Nutr. 2015;54(5):679-89.

5. Morley JE. Defining undernutrition (malnutrition) in older persons. J Nutr Health Aging. 2018;22(3):308-10.

6. Malavolta M, Mocchegiani E. Molecular basis of nutrition and aging: a volume in the molecular nutrition series. Academic Press; 2016. ISBN 9780128018163.

7. Dai L, Mukai H, Lindholm B, Heimburger O, Barany P, Stenvinkel P, Qureshi AR. Clinical global assessment of nutritional status as predictor of mortality in chronic kidney disease patients. PLoS One. 2017;12(12):e0186659.

8. Cirillo M, Laurenzi M, Mancini M, Zanchetti A, Lombardi C, De Santo NG. Low glomerular filtration in the population: prevalence, associated disorders, and awareness. Kidney Int. 2006;70(4):800-6.

9. Duenhas MR, Draibe SA, Avesani CM, Sesso R, Cuppari L. Influence of renal function on spontaneous dietary intake and on nutritional status of chronic renal insufficiency patients. Eur J Clin Nutr. 2003;57(11):1473-8.

10. Topinkova E. Aging, disability and frailty. Ann Nutr Metab. 2008;52(Suppl 1):6-11.

11. Carrero JJ, Aguilera A, Stenvinkel P, Gil F, Selgas R, Lindholm B. Appetite disorders in uremia. J Ren Nutr. 2008;18(1):107-13.

12. Ikizler TA, Hakim RM. Nutrition in end-stage renal disease. Kidney Int. 1996; 50(2):343-57.

13. Schrader E, Grosch E, Bertsch T, Sieber CC, Volkert D. Nutritional and functional status in geriatric day hospital patients - MNA short form versus full MNA. J Nutr Health Aging. 2016;20(9):918-26.

14. Kiesswetter E, Pohlhausen S, Uhlig K, Diekmann R, Lesser S, Heseker $H_{\text {, }}$ Stehle $\mathrm{P}$, Sieber CC, Volkert D. Malnutrition is related to functional impairment in older adults receiving home care. J Nutr Health Aging. 2013; 17(4):345-50.

15. Cruz-Jentoft AJ, Kiesswetter E, Drey M, Sieber CC. Nutrition, frailty, and sarcopenia. Aging Clin Exp Res. 2017;29(1):43-8.

16. Adly NN, Abd-El-Gawad WM, Abou-Hashem RM. Relationship between malnutrition and different fall risk assessment tools in a geriatric in-patient unit. Aging Clin Exp Res. 2020;32(7):1279-87.

17. Correia MI, Waitzberg DL. The impact of malnutrition on morbidity, mortality, length of hospital stay and costs evaluated through a multivariate model analysis. Clin Nutr. 2003;22(3):235-9.

18. Alzahrani SH, Alamri SH. Prevalence of malnutrition and associated factors among hospitalized elderly patients in King Abdulaziz University Hospital, Jeddah, Saudi Arabia. BMC Geriatr. 2017;17(1):136.

19. Michalakis K, Goulis DG, Vazaiou A, Mintziori G, Polymeris A, AbrahamianMichalakis A. Obesity in the ageing man. Metabolism. 2013;62(10):1341-9.

20. Kalish VB. Obesity in older adults. Prim Care. 2016;43(1):137-44.

21. Ouchi N, Parker JL, Lugus JJ, Walsh K. Adipokines in inflammation and metabolic disease. Nat Rev Immunol. 2011;11(2):85-97.

22. Luyckx VA, Tuttle KR, Garcia-Garcia G, Gharbi MB, Heerspink HJL, Johnson DW, Liu Z-H, Massy ZA, Moe O, Nelson RG, et al. Reducing major risk factors for chronic kidney disease. Kidney Int Suppl. 2017;7(2):71-87.

23. MacLaughlin HL, Hall WL, Sanders TAB, Macdougall IC. Risk for chronic kidney disease increases with obesity: health survey for England 2010. Public Health Nutr. 2015;18(18):3349-54.

24. Corsonello A, Tap L, Roller-Wirnsberger R, Wirnsberger G, Zoccali C, Kostka T, Guligowska A, Mattace-Raso F, Gil P, Fuentes LG, et al. Design and methodology of the screening for CKD among older patients across Europe (SCOPE) study: a multicenter cohort observational study. BMC Nephrol. 2018;19(1):260

25. Tonelli M, Riella M. Chronic kidney disease and the ageing population. Lancet. 2014;383(9925):1278-9.

26. Stevens PE, Levin A, Kidney Disease: Improving Global Outcomes Chronic Kidney Disease Guideline Development Work Group M. Evaluation and management of chronic kidney disease: synopsis of the kidney disease: improving global outcomes 2012 clinical practice guideline. Ann Intern Med. 2013;158(1 1):825-30.

27. Stevens PE, O'Donoghue DJ, de Lusignan S, Van Vlymen J, Klebe B, Middleton R, Hague N, New J, Farmer CK. Chronic kidney disease management in the United Kingdom: NEOERICA project results. Kidney Int. 2007:72(1):92-9.

28. Guigoz Y, Vellas B, Garry PJ. Assessing the nutritional status of the elderly: the mini nutritional assessment as part of the geriatric evaluation. Nutr Rev. 1996;54(1 Pt 2):S59-65.

29. Ingwersen S, Raabo E. Improved and more specific bromcresol green methods for the manual and automatic determination of serum albumin. Clin Chim Acta. 1978;88(3):545-50.

30. Schlenker E, Roth SL. Williams' essentials of nutrition and diet therapy revised reprint. 10th ed. United States of America: Elsevier Mosby; 2013.

31. Schaeffner ES, Ebert N, Delanaye P, Frei U, Gaedeke J, Jakob O, Kuhlmann MK, Schuchardt M, Tolle M, Ziebig R, et al. Two novel equations to estimate kidney function in persons aged 70 years or older. Ann Intern Med. 2012;157(7):471-81.

32. Corsonello A, Roller-Wirnsberger R, Di Rosa M, Fabbietti P, Wirnsberger G, Kostka T, Guligowska A, Tap L, Mattace-Raso F, Gil P, et al. Estimated glomerular filtration rate and functional status among older people: a systematic review. Eur J Intern Med. 2018;56:39-48.

33. Levin A, Stevens PE, Bilous RW, Coresh J, De Francisco ALM, De Jong PE, Griffith KE, Hemmelgarn BR, Iseki K, Lamb EJ, et al. Kidney disease: Improving global outcomes (KDIGO) CKD work group. KDIGO 2012 clinical practice guideline for the evaluation and management of chronic kidney disease. Kidney Int Suppl. 2013;3(1):150.

34. Conwell Y, Forbes NT, Cox C, Caine ED. Validation of a measure of physical illness burden at autopsy: the cumulative illness rating scale. J Am Geriatr Soc. 1993;41(1):38-41.

35. IDF Consensus Worldwide Definition of the Metabolic Syndrome. 2017. https://www.idf.org/component/attachments/attachments.html?id=705 \&task=download. Accessed 18 Apr 2020.

36. Noori N, Hosseinpanah F, Nasiri AA, Azizi F. Comparison of overall obesity and abdominal adiposity in predicting chronic kidney disease incidence among adults. J Ren Nutr. 2009;19(3):228-37.

37. Ritchie SA, Connell JM. The link between abdominal obesity, metabolic syndrome and cardiovascular disease. Nutr Metab Cardiovasc Dis. 2007; 17(4):319-26.

38. Fox CS, Massaro JM, Hoffmann U, Pou KM, Maurovich-Horvat P, Liu CY, Vasan RS, Murabito JM, Meigs JB, Cupples LA, et al. Abdominal visceral and subcutaneous adipose tissue compartments: association with metabolic risk factors in the Framingham heart study. Circulation. 2007; 116(1):39-48,

39. Wu Z, Jiang Y, Jia J, He D, Sun P, Li J, Huo Y, Fan F, Zhang Y. Metabolic syndrome is associated with rapid estimated glomerular filtration rate decline in a Chinese community-based population. Diabetes Metab Syndr Obes. 2019;12:2085-93.

40. Thomas G, Sehgal AR, Kashyap SR, Srinivas TR, Kirwan JP, Navaneethan SD. Metabolic syndrome and kidney disease: a systematic review and metaanalysis. Clin J Am Soc Nephrol. 2011;6(10):2364-73.

41. Burton JO, Gray $\amalg$, Webb DR, Davies MJ, Khunti K, Crasto W, Carr SJ, Brunskill NJ. Association of anthropometric obesity measures with chronic kidney disease risk in a non-diabetic patient population. Nephrol Dialysis Transplant. 2011;27(5):1860-6.

42. Lean ME, Han TS, Morrison CE. Waist circumference as a measure for indicating need for weight management. BMJ. 1995;311(6998):158-61.

43. Evans PD, Mclntyre NJ, Fluck RJ, McIntyre CW, Taal MW. Anthropomorphic measurements that include central fat distribution are more closely related with key risk factors than BMI in CKD stage 3. PLoS One. 2012;7(4):e34699.

44. Winter JE, Maclnnis RJ, Wattanapenpaiboon N, Nowson CA. BMI and allcause mortality in older adults: a meta-analysis. Am J Clin Nutr. 2014; 99(4):875-90.

45. Lee CMY, Huxley RR, Wildman RP, Woodward M. Indices of abdominal obesity are better discriminators of cardiovascular risk factors than BMI: a meta-analysis. J Clin Epidemiol. 2008;61 (7):646-53.

46. Correa MM, Thume E, De Oliveira ER, Tomasi E. Performance of the waist-toheight ratio in identifying obesity and predicting non-communicable diseases in the elderly population: a systematic literature review. Arch Gerontol Geriatr. 2016;65:174-82.

47. Ashwell M, Gibson S. Waist-to-height ratio as an indicator of 'early health risk': simpler and more predictive than using a 'matrix' based on BMI and waist circumference. BMJ Open. 2016;6(3):e010159.

48. de Oliveira CC, Roriz AK, Ramos LB, Gomes Neto M. Indicators of adiposity predictors of metabolic syndrome in the elderly. Ann Nutr Metab. 2017; 70(1):9-15. 
49. Obesity: preventing and managing the global epidemic. Report of a WHO consultation. World Health Organ Tech Rep Ser. 2000;894:i-xii, 1-253. ISBN 9241208945.

50. Sánchez-García S, García-Peña C, Duque-López MX, Juárez-Cedillo T, Cortés-Núñez AR, Reyes-Beaman S. Anthropometric measures and nutritional status in a healthy elderly population. BMC Public Health. 2007;7(1):2.

51. Benitez Brito N, Suarez Llanos JP, Fuentes Ferrer M, Oliva Garcia JG, Delgado Brito I, Pereyra-Garcia Castro F, Caracena Castellanos N, Acevedo Rodriguez CX, Palacio Abizanda E. Relationship between midupper arm circumference and body mass index in inpatients. PLoS One. 2016;11(8):e0160480.

52. Arroyo M, Freire M, Ansotegui L, Rocandio AM. Intraobserver error associated with anthropometric measurements made by dietitians. Nutr Hosp. 2010;25(6):1053-6

53. Scallan J, Huxley VH, Korthuis RJ. Pathophysiology of edema formation. In: Capillary fluid exchange: regulation, functions, and pathology. San Rafael: Morgan \& Claypool Life Sciences; 2010.

54. Siddall EC, Radhakrishnan J. The pathophysiology of edema formation in the nephrotic syndrome. Kidney Int. 2012;82(6):635-42.

55. Levey AS, Coresh J. Chronic kidney disease. Lancet. 2012;379(9811): $165-80$.

56. Juby AG, Mager DR. A review of nutrition screening tools used to assess the malnutrition-sarcopenia syndrome (MSS) in the older adult. Clin Nutr ESPEN. 2019;32:8-15.

\section{Publisher's Note}

Springer Nature remains neutral with regard to jurisdictional claims in published maps and institutional affiliations.

Ready to submit your research? Choose BMC and benefit from:

- fast, convenient online submission

- thorough peer review by experienced researchers in your field

- rapid publication on acceptance

- support for research data, including large and complex data types

- gold Open Access which fosters wider collaboration and increased citations

- maximum visibility for your research: over $100 \mathrm{M}$ website views per year

At $\mathrm{BMC}$, research is always in progress.

Learn more biomedcentral.com/submissions 